\title{
BMJ Open Comparison of Outcomes of antibiotic Drugs and Appendectomy (CODA) trial: a protocol for the pragmatic randomised study of appendicitis treatment
}

\author{
Giana H Davidson, ${ }^{1}$ David R Flum, ${ }^{1}$ David A Talan, ${ }^{2}$ Larry G Kessler, ${ }^{3}$ \\ Danielle C Lavallee, ${ }^{1}$ Bonnie J Bizzell, ${ }^{4}$ Farhood Farjah, ${ }^{1}$ Skye D Stewart, ${ }^{1}$ \\ Anusha Krishnadasan, ${ }^{2}$ Erin E Carney, ${ }^{1}$ Erika M Wolff, ${ }^{1}$ Bryan A Comstock, ${ }^{5}$ \\ Sarah E Monsell, ${ }^{5}$ Patrick J Heagerty, ${ }^{5}$ Annie P Ehlers, ${ }^{1}$ Daniel A DeUgarte, ${ }^{6}$ \\ Amy H Kaji, ${ }^{7}$ Heather L Evans, ${ }^{8}$ Julianna T Yu, ${ }^{9}$ Katherine A Mandell, ${ }^{10}$ \\ Ian C Doten, ${ }^{11}$ Kevin S Clive, ${ }^{12}$ Karen M McGrane, ${ }^{13}$ Brandon C Tudor, ${ }^{14}$ \\ Careen S Foster, ${ }^{15}$ Darin J Saltzman, ${ }^{16}$ Richard C Thirlby, ${ }^{17}$ Erin O Lange, ${ }^{1}$ \\ Amber K Sabbatini, ${ }^{18}$ Gregory J Moran ${ }^{2}$
}

To cite: Davidson GH, Flum DR, Talan DA, et al. Comparison of Outcomes of antibiotic Drugs and Appendectomy (CODA) trial: a protocol for the pragmatic randomised study of appendicitis treatment. BMJ Open 2017;7:e016117. doi:10.1136/ bmjopen-2017-016117

- Prepublication history for this paper is available online. To view please visit the journal online (http://dx.doi.org/10. 1136/bmjopen-2017-016117).

Received 27 January 2017 Revised 6 October 2017 Accepted 12 October 2017

CrossMark

For numbered affiliations see end of article.

Correspondence to Dr Giana H Davidson; ghd@uw.edu

\section{ABSTRACT}

Introduction Several European studies suggest that some patients with appendicitis can be treated safely with antibiotics. A portion of patients eventually undergo appendectomy within a year, with $10 \%-15 \%$ failing to respond in the initial period and a similar additional proportion with suspected recurrent episodes requiring appendectomy. Nearly all patients with appendicitis in the USA are still treated with surgery. A rigorous comparative effectiveness trial in the USA that is sufficiently large and pragmatic to incorporate usual variations in care and measures the patient experience is needed to determine whether antibiotics are as good as appendectomy.

Objectives The Comparing Outcomes of Antibiotic Drugs and Appendectomy (CODA) trial for acute appendicitis aims to determine whether the antibiotic treatment strategy is non-inferior to appendectomy.

Methods/Analysis CODA is a randomised, pragmatic non-inferiority trial that aims to recruit 1552 Englishspeaking and Spanish-speaking adults with imagingconfirmed appendicitis. Participants are randomised to appendectomy or 10 days of antibiotics (including an option for complete outpatient therapy). A total of 500 patients who decline randomisation but consent to followup will be included in a parallel observational cohort. The primary analytic outcome is quality of life (measured by the EuroQol five dimension index) at 4 weeks. Clinical adverse events, rate of eventual appendectomy, decisional regret, return to work/school, work productivity and healthcare utilisation will be compared. Planned exploratory analyses will identify subpopulations that may have a differential risk of eventual appendectomy in the antibiotic treatment arm.

Ethics and dissemination This trial was approved by the University of Washington's Human Subjects Division. Results from this trial will be presented in international conferences and published in peer-reviewed journals. Trial registration number NCT02800785.
Strengths and limitations of this study

This trial will evaluate the comparative effectiveness of antibiotics and appendectomy for appendicitis based on a comprehensive assessment of impact, including the full range of clinical outcomes and patient-reported outcomes that matter most to patients.

- This pragmatic trial was designed to account for the diverse aspects of the population, practice settings and practices in the USA.

- This study builds on the successful experience of clinicians to manage patients with potentially serious infections as outpatients using risk stratification and long-acting parenteral antibiotics.

\section{INTRODUCTION}

Acute appendicitis is the most common reason for an urgent abdominal operation, with a lifetime incidence of $7 \%-15 \% .{ }^{1}$ Each year, nearly 300000 Americans are hospitalised for appendicitis at a cost of US $\$ 7.8$ billion. $^{23}$ While appendectomy has been the treatment of choice for 120 years, the successful use of antibiotics was reported both in a series of over 500 patients treated with streptomycin in the 1950s and later in submariners who did not have access to surgical teams. ${ }^{45}$ As anaesthesia and surgical safety improved throughout the 20th century, the antibiotics treatment strategy was relegated to patients with disease severe enough (eg, phlegmon at the cecum, abscess) that surgeons felt there was a higher risk for surgical complications or the need for a more extensive procedure. 
Based on these successes with an antibiotic strategy, in the 1990s, European investigators began challenging the notion that surgery was the best approach to treat acute 'uncomplicated' appendicitis with a series of randomised trials comparing antibiotics and appendectomy. ${ }^{4-10}$ A recent meta-analysis of six randomised trials including 1724 randomised adult patients concluded that there was a high level of efficacy $(91 \%$ success in the short term with $71 \%$ appendectomy-free by 1 year), less pain and a quicker return to work in the antibiotic arm. ${ }^{11}$ The largest, most rigorous and recent trial found a lower rate of postinterventional complications (reported as clinical wound infections, incisional hernia, abdominal pain or obstructive symptoms) in the antibiotics group requiring intervention when compared with those having open surgical procedures. ${ }^{12}$ However, in addition to the potential for recurrence of appendicitis, a small proportion of patients treated with antibiotics likely had a neoplasm that would have been incidentally identified had they undergone appendectomy. A recent meta-analysis reported incidental appendiceal neoplasm in 5 of 843 $(0.59 \%)$ patients undergoing surgery. ${ }^{11}$ The meta-analysis overall concluded that laparoscopic appendectomy remains the usual treatment for appendicitis and there is a "poor evidence base overall with numerous areas of bias', limiting the use of the data for decision making.

The limitations of the existing data regarding antibiotics as a primary treatment for acute appendicitis have been systematically reviewed. ${ }^{13}$ Most studies had small sample sizes; several did not have standardised imaging for diagnosing appendicitis, leading to inclusion of patients who likely had 'complicated' appendicitis and patients without appendicitis; inexact and subjective outcome definitions and operation/reoperation criteria were used; there were limited or no laparoscopic options for surgery and, in some cases, inadequate antibiotic regimens allowed; and most had short follow-up (no studies reported following patients beyond 1 year) ${ }^{13}$ While some studies evaluated outcomes including general pain scores and use of narcotic pain medication, no study used a validated patient-reported outcome (PRO) tool to measure the patient's experience in a standardised fashion. Other important outcomes to patients such as impact on work and school productivity, lingering symptoms, decisional regret and healthcare burden (such as emergency room care or future imaging) were not included in prior studies. Furthermore, prior studies regimented care in ways that are not consistent with care in the USA, such as requiring several days of inhospital convalescence. These limitations may explain the infrequent use of antibiotics as the primary treatment for appendicitis in the USA. ${ }^{14}$

In addition to the need to address these limitations, there are additional, unresolved questions that make a larger, more definitive study of this treatment question important. First, there may be important subgroups of people with acute appendicitis who experience the treatment differentially. These might include older patients, who are at higher risk for surgical complications, those with possible appendiceal perforation detected on imaging or those with an appendicolith. The association between appendicolith and worse outcomes with antibiotics is unclear. Appendicoliths are found in up to $20 \%$ of appendicitis cases; a similar proportion is also described in autopsy studies of normal appendices. ${ }^{15}$ In several paediatric studies and at least one adult study, appendicolith seemed to be associated with eventual appendectomy; however, since many trials did not include standardised imaging or criteria for requiring appendectomy following antibiotic therapy for appendicitis, it is unclear if the presence of an appendicolith actually confers a greater risk. ${ }^{16}{ }^{17}$ There is currently no standard definition of 'complicated' disease. In the USA, usual care for appendiceal abscess or phlegmon (inflammation so significant that surgeons are concerned for associated surgical morbidity) is antibiotics with consideration for interval appendectomy. Optimal treatment strategies for preoperative radiographic findings of appendiceal perforation are an area of controversy. The use of radiologic imaging to accurately determine perforation is limited; in prior randomised trials, patients with perforation were likely to have been inadvertently included due to a lack of imaging. ${ }^{18}$ Finally, the European studies mandated the use of inpatient antibiotics at a time when there was a growing use of outpatient antibiotic regimens for similar conditions, such as acute diverticulitis. ${ }^{19-21}$ A recently completed, pilot randomised trial in the USA found that 14 of 15 adults randomised to antibiotics could successfully be discharged from the emergency department (ED) and receive all their care as outpatients, resolving their symptoms of acute appendicitis. ${ }^{22}$ One of the remaining questions is whether this total outpatient approach to antibiotics would be as good as appendectomy in usual practice.

Given these evidence gaps it remains to be determined if, from the patient's perspective, the antibiotic treatment approach is similar, definitively not worse, and perhaps even superior than the standard treatment of appendectomy. The Comparing Outcomes of Antibiotic Drugs and Appendectomy (CODA) trial was designed to address this question and inform decision-making, focusing on commonly used surgical strategies and a range of antibiotic strategies, including total outpatient therapy, across a broad range of practice environments and a heterogeneous group of patients. These questions provide strong motivation for a pragmatic trial of antibiotics for acute appendicitis.

\section{TRIAL DESIGN}

Stakeholder input in design, informed consent and protocol A central feature of the CODA trial is its engagement of stakeholders in study conception, design, and implementation of the trial. ${ }^{23}$ The Stakeholder Coordinating Center (SCC), established as a formal core within the study infrastructure, facilitates all engagement activities. The SCC engages representatives from the patient 
population of interest (those at risk for or who have had appendicitis), clinicians who are involved in appendicitis treatment (including emergency physicians, nurses and surgeons), leaders of professional societies (American College of Surgeons and American College of Emergency Physicians), representatives of Accountable Care Organizations, policy-makers, insurers and payers, researchers and leaders from large self-insured employers. Specific areas of protocol development informed by SCC included selecting primary and secondary outcomes. In addition to the routine clinical metrics that are assessed in any study of appendicitis treatment, other outcome measures important to patients (anxiety, quality of life (QoL), time away from work, out-of-pocket expenses) and employers (time away from work and productivity at work) were included. Stakeholder input was particularly helpful in determining the primary analytic outcome, helping weigh the prior evidence showing no difference in rates of complications with an outcome metric that would 'sum up' the impact of both treatments on the care experience of patients.

Because appendectomy was considered the standard and nearly universal therapy in the USA, advisors recommended a study that considered the non-inferiority of the antibiotics-first strategy. As one advisor said, 'the burden of proof is on the antibiotics treatment approach to demonstrate that it is as good as appendectomy' (or not inferior by more than a small margin). Advisors also favoured a non-inferiority framework because the larger size required for this design would also allow for multiple planned subgroup analyses for patient groups of interest and the possibility that superiority of the PRO measure might be demonstrated. Lastly, advisors suggested a parallel observational cohort to assess for potential selection bias for patients who declined randomisation.

Patient advisors with an experience of incidentally identified neoplasm at the time of appendectomy helped modify the inclusion criteria (excluding all patients with suggestion of mass of the appendix on imaging) and consent form (adding language to make sure that patients were informed about this risk, estimated to be $0.6 \%$ ) and directed a change in the protocol (those with lingering symptoms in the antibiotics group would be directed to follow-up visits and usual care diagnostic evaluations to rule out a neoplasm).

\section{Study aims and hypothesis}

The aims of the study are to compare PROs and clinical outcomes in patients randomised to antibiotics or appendectomy. We hypothesise that antibiotics are non-inferior to appendectomy for PROs and that there are subgroups with better outcomes (clinical and patient reported) with either treatment. A second set of aims is to perform subpopulation analyses for patients with appendicolith and imaging correlates that may indicate higher risk of requiring appendectomy following initiation of antibiotic therapy, advanced age, sex, comorbid conditions and insurance status.

\section{Study population}

The study population includes consecutively presenting English-speaking or Spanish-speaking adults (age $\geq 18$ years) with clinically suspected and imaging-confirmed acute appendicitis who present at study site hospital EDs in several states.

\section{Exclusion criteria}

- Inability to participate in follow-up (ie, incarcerated, travel without access to phone, email)

- Contraindication to one of the study treatment arms:

- Septic shock (evidence of severe sepsis or septic shock includes new presumed sepsis-related organ dysfunction, elevated lactate and/or fluid unresponsive hypotension)

- Phlegmon for which surgery would not be recommended or diffuse peritonitis for which antibiotics alone would not be recommended

- Imaging findings of walled off abscess and/or free air

- Appendiceal soft-tissue mass concerning for malignancy

- Other conditions precluding study involvement:

- Uncompensated liver failure

- Inflammatory bowel disease requiring active medical treatment (eg, Crohn's, ulcerative colitis)

- Pregnancy or expectation of becoming pregnant in the 30 days following baseline/screening.

- Surgical implant (eg, left ventricular assist device, peritoneal dialysis)

- Malignancy requiring active treatment (eg, chemotherapy)

- Immunodeficiency (eg, AIDS)

- Another infection currently treated with systemic antibiotics

- Concurrent illness that would otherwise mandate inpatient hospitalisation

- Severe allergy or reaction to all proposed antibiotics

- Abdominal or pelvic surgery in the past 30 days.

Of note, patients with radiologic diagnosis of appendicolith and/or imaging concerning for appendiceal perforation or phlegmon are included if they do not meet the above exclusion criteria and are otherwise eligible.

\section{Recruitment}

All patients presenting to the ED with concern for appendicitis are screened by study coordinators ( 7 days a week, at least 18 hours per day) based on alerts from clinicians, staff and screening of ED logs. Patients are identified as potential study candidates based on eligibility criteria collected as part of standard care, including confirmatory diagnostic imaging (CT, ultrasound and/ or MRI). A research coordinator and a representative from the clinical team confirm the patient's eligibility for the study. A research team member approaches all eligible patients and invites them to view a less than 10 min standardised informed decision-making video providing standard information about appendicitis and 
Table 1 Participant assessment schedule

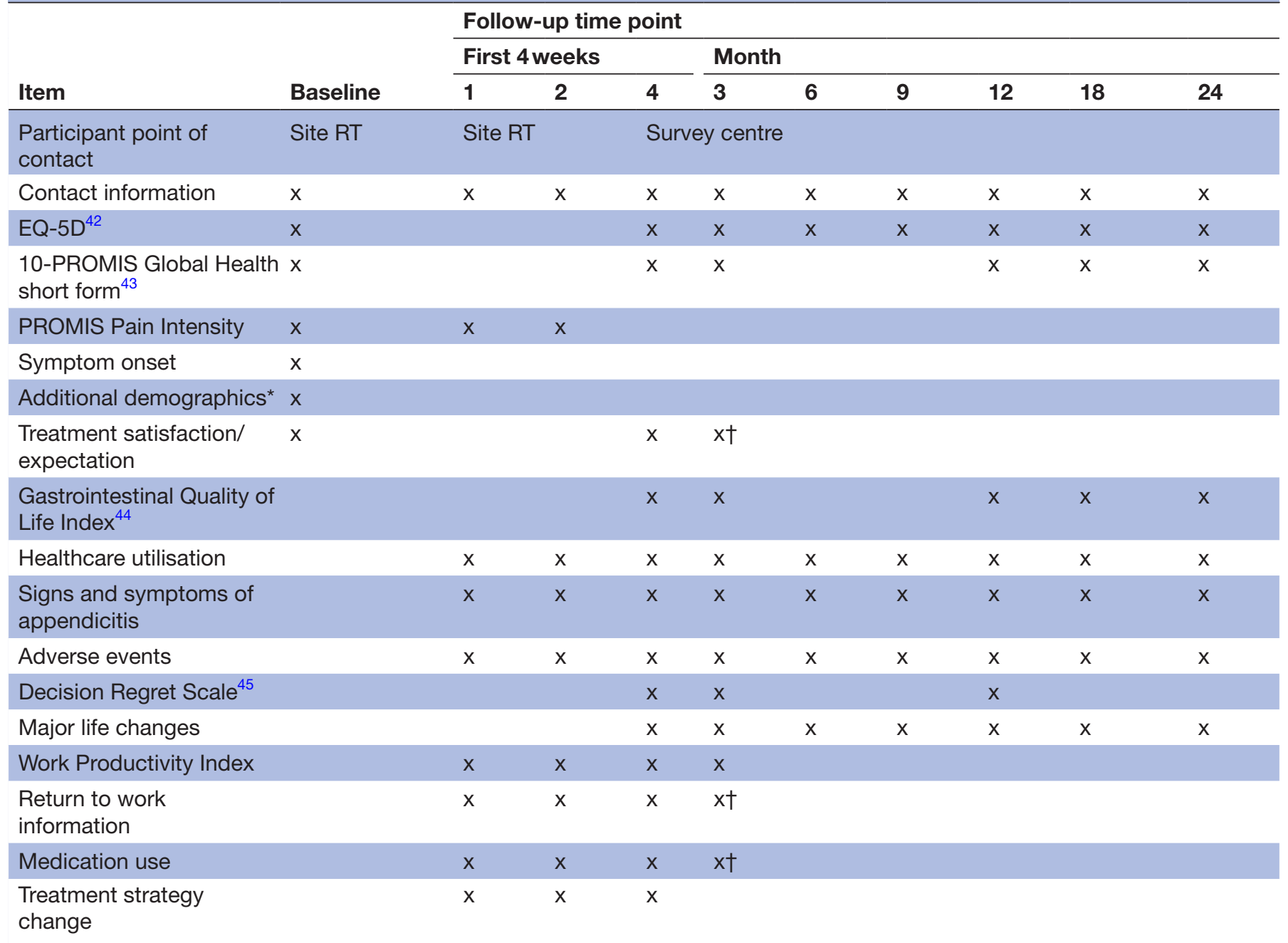

*Includes the following topics: demographics and gender identity, caregiver role, instrumental support, employment/student status, income, pain catastrophising, health literacy, social support, confidence in treatment success and trust in healthcare.

†Only asked if the 1-month results have not normalised.

EQ-5D, EuroQol five dimension; PROMIS, patient-reported outcomes measurement information system; RT, research team.

the different treatment options (offered in English and Spanish versions, https://www.youtube.com/playlist?list= PLQUQ6jdR0MPaq-a8CvSdhVwnuYzNKF9tu).

Participants who decline randomisation are asked to participate in the observational cohort (with similar baseline and follow-up measures as participants in the randomised controlled trial). All patients are asked for permission to be followed through passive electronic medical record (EMR) review.

\section{Participant follow-up assessment}

Participants are contacted 24-48hours after discharge by a member of the research team to answer any questions about the study and review the survey protocol (see table 1). Participants are then contacted by phone by site research coordinators 1 and 2 weeks after enrolment for study assessments. Data collected through the 2-week assessment are entered by site research coordinators into a REDCap database, which is managed by the University of Washington (UW) data coordinating centre (DCC). ${ }^{24}$
Starting with the week 4 assessment, corresponding to our primary endpoint assessment, participants are contacted by phone, mail or email by the UW Survey Center to complete the remaining study assessments (at $3,6,9,12,18$ and 24 month surveys). The UW Survey Center uses the DatStat survey platform (DatStat, Seattle, Washington, USA) to create individualised outreach plans that optimise survey completion rates. Outreach methods are modified to accommodate a participant's preferred mode of contact (email, mail, phone) as well as time of day for contact (if by phone). If a participant requests to speak with a medical provider or has concerning medical symptoms reported to the research team, the clinical team via the surgical site lead is contacted to call the participant for further follow-up.

The DCC performs early quality assurance checks by running REDCap data quality reports. These reports identify missing values for required fields, incorrect data type, range checks, outliers, hidden fields that contain values 
and multiple-choice fields with invalid values. Values that need to be corrected are brought to the attention of the research staff at that site.

\section{Study arms}

\section{Antibiotics therapy arm}

Patients in the antibiotics treatment arm receive a minimum of 24 hours of treatment using an intravenous antibiotic formulation (administered in q8, q12 or q24 hour regimens) followed by oral antibiotics for a total of a 10-day antibiotic course. Patients are offered a treatment regimen of antibiotics based on guidelines published jointly by the Surgical Infection Society (SIS) and the Infectious Disease Society of America (IDSA) for intravenous antibiotics ${ }^{25}$ and oral antibiotics based on in vitro activity against aerobic and anaerobic Gram-negative bacteria, practical experience with oral antibiotic regimens used to treat diverticulitis and IDSA/SIS guidelines. The first dose of antibiotics is given in the $\mathrm{ED}$ at the time of diagnosis of appendicitis, and a total outpatient regimen of antibiotics is an option for patients meeting ED discharge criteria. Antibiotics are procured from the pharmacy by the patient as per usual clinical care.

Appendectomy is recommended only if there is development of diffuse peritonitis, development of septic shock ${ }^{26}$ and/or worsening signs and symptoms of appendicitis after 48 hours. The decision to perform an appendectomy in participants randomised to antibiotics is made by the treating surgeon after consultation with the study clinical research lead to confirm that the above criteria have been satisfied.

Standard discharge criteria are applied to those treated in the ED and those who are admitted, and the criteria include tolerance of liquids, adequate pain control and improving clinical condition. All participants are contacted at 24-48 hours by the research coordinator to review the study protocol for follow-up assessments.

Follow-up with the clinical team is per usual care at each institution. Participants in the antibiotics arm who return to any of the study sites during the follow-up period with recurrent appendicitis are not rerandomised but are offered the choice of either appendectomy or another antibiotic course, if treating surgeon agrees that their recurrence can be treated with either option.

\section{Appendectomy therapy arm}

All patients randomised to appendectomy receive preoperative antibiotics per hospital standards for surgical infection prevention protocols. Appendectomy is performed by an open or laparoscopic approach, depending on patient and surgeon preference.

\section{Blinding and randomisation}

This is an unblinded study as patients will know if they were randomised to appendectomy or antibiotics. A separate DCC at UW generates and maintains randomisation lists for each practice site. Using block randomisation optimises the chances of equal numbers of subjects being randomised to each treatment arm, and that treatment is balanced at periodic enrolment intervals. Randomisation is further stratified by the presence of appendicolith. All other subgroups of interest will be sufficiently large such that the risk of a meaningful imbalance in treatment groups by chance is unlikely. A web-based portal provides the randomised treatment assignment.

\section{Outcomes and measures}

The primary outcome for the CODA trial is the EuroQol five dimension (EQ-5D) index reported 4 weeks after randomisation. In addition, important clinical outcomes include major complications and resolution of symptoms by 4 weeks; eventual appendectomy (due to failure in clinical improvement, progression of disease severity or due to recurrent appendicitis), pain, narcotic use, recurrent episodes of appendicitis, ED visits for abdominal pain/ repeat imaging, need for more complicated surgical procedure including laparoscopic converted to open appendectomy and ileocecectomy, rates of perforation and rates of future small bowel obstructions and hernia development are collected and will be reported through 2 years. Complications in both treatment groups are tracked and adjudicated by an independent safety monitor to determine their relation to the disease and treatment. Secondary PROs include a measure of decisional regret, anxiety, additional QoL measures (PROMIS-Global, Gastrointestinal Quality of Life Index), days missed from work or school, time in healthcare, measures of caregiver burden and out-of-pocket expenses.

\section{Sample size}

The sample size was calculated based on the difference in EQ-5D between the two treatment interventions (table 2). The EQ-5D QoL index ranges from 0 (worst QoL) to 1 (highest QoL), where anchor-based methods have shown that the minimally clinically important difference ranges $5 \%-10 \% .{ }^{27}$ On the basis of data from a prior study of appendectomy with EQ-5D scores at 12 weeks, ${ }^{28}$ we estimate that the average EQ-5D for the participants randomised to appendectomy will be 0.90 with an SD of 0.12. To assess QoL differences between interventions, a total of 1552 patients will be enrolled, assuming a $90 \%$ follow-up at 4 weeks. This will give the study very high power $(>99 \%)$ to rule out an EQ-5D difference between

Table 2 Statistical power to declare non-inferiority on patient-reported quality of life, overall and by subgroup (non-inferiority margin, $\mathrm{M}=-5 \%$, one-sided alpha=0.025)

\begin{tabular}{lllll}
\hline $\begin{array}{l}\text { Treatment } \\
\text { difference, }\end{array}$ & \multicolumn{3}{l}{ Overall (\%) } & \multicolumn{3}{c}{ Subgroups (\%) } \\
\cline { 2 - 5 } $\boldsymbol{\Delta}(\%)$ & $\mathrm{n}=\mathbf{1 5 5 2}$ & $\mathrm{n}=\mathbf{2 5 0}$ & $\mathbf{n = 4 0 0}$ & $\mathrm{n}=\mathbf{5 0 0}$ \\
\hline-3 & 82.6 & - & - & - \\
-2 & 99.4 & - & 57.1 & 67.9 \\
-1 & 100 & 62.4 & 83.8 & 91.4 \\
0 & 100 & 83.0 & 96.4 & 98.8 \\
\hline
\end{tabular}




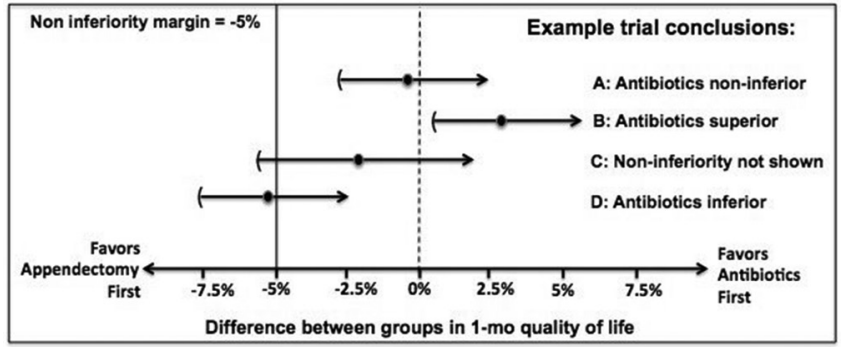

Figure 1 Example study conclusions in the Comparing Outcomes of Antibiotic Drugs and Appendectomy trial. There are four possible study conclusions. (A) The observed treatment effect (black circle) of antibiotics is almost zero and the $97.5 \%$ one-sided $\mathrm{Cl}$ (arrow) does not overlap the non-inferiority margin of $-5 \%$, indicating that antibiotics is a non-interior strategy. (B) The observed treatment effect of antibiotics is more than $2.5 \%$ better than appendectomy and the $\mathrm{Cl}$ does not include 0 , indicating that antibiotics are superior. (C) The observed treatment effect of antibiotics is $2.5 \%$ worse than appendectomy but the $\mathrm{Cl}$ includes $-5 \%$, so non-inferiority cannot be claims. (D) The observed treatment effect of antibiotics is more than $5 \%$ worse than appendectomy, indicating that antibiotics are inferior.

groups as small as 5\% (if treatment differences of $0 \%-2 \%$ are observed) and $80 \%$ power if a treatment difference of $3 \%$ is observed. $^{22}$

On the basis of pilot data and stakeholder engagement, we estimate a randomisation rate of $30 \%$ of all potential patients. Based on current appendectomy volume at the hospitals participating in the trial, recruitment is planned for 3 years with potential for extension through 4 years.

\section{Statistical analysis}

We will assess the EQ-5D at 4 weeks, using a linear regression model that adjusts for an indicator of randomised treatment group assignment and for all factors used to stratify randomisation (ie, recruitment site, presence of appendicolith). As recommended by the US Food and Drug Administration guidelines on clinical trial design, the estimated treatment effect and $97.5 \%$ one-sided CI will be compared with the non-inferiority margin $(\mathrm{M}=-5 \%) \cdot{ }^{29-32}$ We will conclude that antibiotics are non-inferior to appendectomy if the entire $97.5 \%$ one-sided CI is greater than $\mathrm{M}$, as in example scenario A (figure 1). This is equivalent to a one-sided (alpha=0.025) test of the null hypothesis $\mathrm{H}_{0}: \Delta \leq-5 \%$, for which $\Delta$ represents the difference in mean EQ-5D at 4 weeks comparing antibiotics-first to appendectomy-first treatment assignment. If the null hypothesis of $\mathrm{H}_{0}: \Delta \leq-5 \%$ is rejected at the final evaluation, then we will conduct a test of superiority to determine the level of statistical evidence supporting an alternative hypothesis $\mathrm{H}_{\mathrm{A}}: \Delta>0 \%$ (ie, scenario $\mathrm{B}$ of figure 1).

Important clinical endpoints (30-day major complications, days until resolution of symptoms, rates of perforated appendicitis, extent of operation and surgical complications, complications associated with antibiotics, hospital days, number of days using antibiotics beyond the initial treatment, clinic visits and caregiver/patient 'time in healthcare') will also be compared between intention-to-treat (ITT) groups using regression models appropriate to each endpoint (eg, linear, logistic, Poisson or Cox proportional hazards regression models), along with a similar non-inferiority framework.

\section{Secondary analyses}

We aim to include a heterogeneous population of patients and healthcare settings and plan to explore differences in treatment outcomes across subgroups of interest, including those with appendicolith, people with specific imaging findings including possible appendiceal perforation, those in different age groups (18-64 or $\geq 65$ ) and sex and those whose outcomes may vary due to differences in work and insurance status, comorbidities or social support. We will evaluate difference in treatment effectiveness based on modality of receipt of antibiotics (all outpatient vs inpatient/outpatient). We will separately assess treatment effect heterogeneity by adding to the primary outcome model an interaction term between the categorical subgroup variable of interest and the indicator of treatment. We will use a global likelihood ratio test to examine if the treatment effect differs between key subgroups of interest.

An ITT approach will be applied in the primary analysis. We will conduct a secondary as-treated analysis of the primary outcome measure that appropriately accounts for patient-level or provider-level characteristics found to be differentially represented among patients who start in the antibiotics arm and who undergo appendectomy before 24 hours of treatment or patients who are randomised to appendectomy but refuse the procedure and continue on antibiotics. We will consider a two-stage approach for this as-treated analysis: (1) to identify subgroups that are likely to require appendectomy and therefore should not be considered good candidates for treatment with antibiotics as primary treatment strategy and (2) to estimate the complier average causal effect, which seeks to compare the outcomes of patients treated successfully in the antibiotic treatment arm (ie, did not ultimately have surgery) with patients randomised to the appendectomy arm who are similar in their expected compliance to assigned treatment. ${ }^{33-35}$ We will use a maximum-likelihood mixture modelling approach to identify the optimal comparison group from the control arm for observed compliers in the intervention arm. Secondary analyses of the primary outcome measures will include examining the entire trajectory of EQ-5D QoL measurements for each patient using linear mixed-effects models for longitudinal data. ${ }^{36}$ Finally, a composite outcome metric (symptom resolution without complication) was used in the recently completed pilot trial and will be included as an exploratory measure. ${ }^{22}$ Because the composite outcome includes only clinical domains, and is relevant to both treatment groups, this may be a helpful measure for clinicians considering the two treatments. 


\section{Data safety and monitoring}

\section{Event reporting}

Death, life-threatening events and rehospitalisation (other than for treatment of appendicitis) are classified as serious adverse events (SAEs). Morbidity events (using modified definitions from the National Surgical Quality Improvement Program (NSQIP) to accommodate non-operative care) are considered adverse events (AEs). AEs, SAEs and appendectomy after starting antibiotic treatment are identified through three approaches; EMR review, patient surveys and ad hoc reporting by any research or care team member. All SAEs are adjudicated by an independent safety monitor. SAEs and AEs are reviewed by the data and safety monitoring board (DSMB) biannually (with the exception of death, which is reported to the DSMB within 24 hours). An independent DSMB reviews the accruing data to (1) ensure that study conduct, enrolment and patient follow-up is adequate; (2) ensure that there are no serious safety concerns and (3) assess evidence related to patient-reported QoL. The analysis of accruing data is completed by the DCC, and interim analysis is presented to the DSMB with the primary goal of monitoring safety outcomes by randomisation group. Interim monitoring for $\mathrm{SAE}$ and $\mathrm{AE}$ will focus on the first 4 weeks of follow-up. The DSMB will conduct interim analyses at 12, 24 and 36 months.

The CODA trial does not include a stopping rule if non-inferiority is met before complete accrual or if it is determined that non-inferiority cannot be demonstrated in interim analyses. We are not employing a stopping rule because there are important secondary outcomes (eg, rate of eventual appendectomy, complications, subgroup analysis) and understudied subgroups that require full enrolment.

\section{DISCUSSION}

Prior trials randomising patients with appendicitis to antibiotics compared with appendectomy focused on disease cure, with the primary outcome being the rate of appendectomy among antibiotic-treated participants. Previous studies of more than 800 participants randomised to antibiotics suggested that the treatment did not increase the rate of complications and offered as high as a $75 \%$ chance of avoiding appendectomy within a year. ${ }^{6-9} 1237$ What remains to be evaluated is the comparative effectiveness of the two candidate treatments based on a comprehensive assessment of impact, including the full range of clinical outcomes and PROs that matter most to patients. CODA's pragmatic design aims to evaluate antibiotics in a heterogeneous population and practice settings in a large randomised trial, with a parallel observational cohort to assess selection bias. One of the greatest novelties of the CODA trial is its patient centredness, demonstrated both by the engagement of patients and other stakeholders as partners in selecting the topic, designing the proposal, developing the protocol and overseeing operations and in the selection of a QoL endpoint for the primary analysis.
CODA was designed to directly inform patient and clinician decision making in the community, and several pragmatic features were added to make sure it accounted for the diverse aspects of the population, practice settings and practices in the USA. As a pragmatic trial, CODA has limited exclusion criteria and incorporates the many ways clinical care is delivered across sites of practice. The protocol allows patients in either study arm to leave the healthcare setting as soon as standard discharge criteria are met, including the possibility of completely outpatient care. CODA takes place in diverse study sites (academic, private, public, community and county hospitals) with patients from a wide range of demographic and socioeconomic characteristics, including both Spanish and English speakers. This enhances the generalisability of the findings, but may compromise study fidelity if patients in any one group have differential treatment preferences or prove more difficult to contact for follow-up. A downside to this approach is that, by including nearly all patients with appendicitis (including those with appendicolith and radiographic findings of perforation who may be at higher risk for requiring an appendectomy) and those undergoing total outpatient antibiotics (which clinicians have less experience with), there is a risk of subgroups with very different outcomes from the broader population and a skewing of the average study results. Using Thorpe's PRagmatic-Explanatory Continuum Indicator Summary rubric for pragmatic trials, the proposed study is considered highly pragmatic, intended to improve the generalisation and precision of decision-making beyond the prior randomised studies. ${ }^{38}$

The results from the European trials of antibiotics have not significantly changed care delivery in the USA and have been met with resistance, in part due to the evidence gaps cited earlier and concern about the fate of patients with recurrent disease. ${ }^{39}$ American patients may also have different expectations and resources that influence perception of treatment success and satisfaction with treatments. One particular protocol component of the European trials that may make them less applicable to the US experience is that prior studies all required an inhospital convalescence for a fixed period of time for both treatment arms that is double the length of stay that the average US patient experiences. CODA builds on the successful experience of emergency medicine clinicians to manage patients with potentially serious infections as outpatients using risk stratification and long-acting parenteral antibiotics (eg, diverticulitis), and its effectiveness will be tested in different practice settings and populations. This novel treatment alternative offers avoidance of hospital admission and may substantially reduce costs compared with surgical treatment,

Stakeholder input is a key component of the emerging field of patient-centred outcomes research. However, including several types of stakeholders (patients, physicians, payers and purchasers) does not always result in consensus. The selection of an appropriate analytic outcome for the trial was an example. While prior 
studies focused on clinical outcome (eg, rates of appendectomy and surgical complications), patient advisors recognised that these outcome measures are specific to only one treatment arm (and to people treated with antibiotics who proceed to appendectomy) and that standardised measurements of QoL would be applicable to both and had yet to be rigorously assessed. The EQ-5D has been used in prior studies of appendectomy, but never in comparisons of these two treatments. ${ }^{28}$ Using the EQ-5D as a primary outcome measure was highly relevant to many, but not all, patients. There is a possibility that the primary analytic outcome analysis (non-inferiority of the EQ-5D) could be positive, but other outcome domains might not be aligned. For this reason, multiple secondary analyses and exploratory endpoints have been selected a priori. Evidence in the field of decision-making suggests that patients want information on multiple domains, but we recognise that multiple outcome domains may also add confusion to interpretation of results and implementation in future practice.

As in all trials, patients are not required to stay in the treatment arms they are assigned to (non-adherence or crossover); for example, select patients in the antibiotics arm might not be willing to receive 24 hours of antibiotics and opt for an appendectomy despite not meeting clinical trial protocol recommendations or patients randomised to appendectomy might refuse surgery. While the main analytic approach is an ITT framework, careful as-treated and secondary data analyses may be helpful in accounting for such non-adherence/crossover. ${ }^{40}$ Detry and Lewis recommend both an ITT and a careful as-treated analysis to address crossovers in non-inferiority trials where non-adherence or crossover is present. ${ }^{41} \mathrm{~A}$ simple as-treated analysis is problematic because of potential differences in demographic or clinical characteristics that introduce bias in as-treated group comparisons. Our analytic approach proposed involves a two-stage as-treated analysis and potentially will yield conclusions that differ from ITT analysis. However, the ITT results will be considered the primary analysis and are robustly valid since they only depend on randomisation and do not depend on model assumptions required for observational comparisons. ${ }^{41}$

CODA began recruitment in the summer/fall of 2016 with eight hospitals in Washington and California with additional hospitals across the United States planned to begin recruitment in 2017. It is possible that not all clinical sites will continue to contribute patients throughout the entire recruitment period (projected to be 3-4 years). Substudies and ancillary studies are being proposed to focus on biomarkers, economic analysis, longer-term results and other predictors of outcome.

In conclusion, the CODA trial was designed to address critical knowledge gaps related to the treatment of appendicitis with antibiotics compared with appendectomy. CODA's stakeholder-informed design and operations, pragmatic design and inclusion of an innovative approach to outpatient antibiotics aim to inform choices in care for this common condition, and planned subgroup analyses allow for improved decision making.

\section{Author affiliations}

${ }^{1}$ Department of Surgery, University of Washington, Seattle, Washington, USA ${ }^{2}$ Department of Emergency Medicine, Olive-View UCLA Medical Center, Sylmar, California, USA

${ }^{3}$ Department of Health Services, University of Washington, Seattle, Washington, USA ${ }^{4}$ The Comparative Effectiveness Research Translation Network, CODA Chair, Patient Advisory Group, Seattle, Washington, USA

${ }^{5}$ Department of Biostatistics, University of Washington, Seattle, Washington, USA ${ }^{6}$ Department of Surgery, Harbor-UCLA Medical Center, Torrance, Califorina, USA ${ }^{7}$ Department of Emergency Medicine, Harbor-UCLA Medical Center, Torrance, California, USA

${ }^{8}$ Department of Surgery, Harborview Medical Center, Seattle, Washington, USA ${ }^{9}$ Department of Emergency, Virginia Mason Medical Center, Seattle, Washington, USA

${ }^{10}$ Department of Surgery, Swedish Medical Center-First Hill, Seattle, Washington, USA

${ }^{11}$ Department of Emergency Medicine, Swedish Medical Center-First Hill, Seattle, Washington, USA

${ }^{12}$ Department of Surgery, Madigan Army Medical Center, Fort Lewis, Washington, USA

${ }^{13}$ Department of Emergency Medicine, Madigan Army Medical Center, Fort Lewis, Washington, USA

${ }^{14}$ Department of Emergency Medicine, Providence Regional Medical Center, Everett, Washington, USA

${ }^{15}$ Department of Trauma and Acute Care Surgery, Providence Regional Medical Center, Everett, WA, USA

${ }^{16}$ Department of Surgery, Olive-View UCLA Medical Center, Sylmar, California, USA

${ }^{17}$ Department of Surgery, Virginia Mason Medical Center, Seattle, Washington, USA

${ }^{18}$ Department of Emergency Medicine, Harborview Medical Center, Seattle,

Washington, USA

Contributors DRF conceived of the study and is the primary grant holder. GHD, DRF, DAT, LGK, DCL, AK, SDS, BAC, PJH, APE and GJM initiated the study design. DAT, LGK, DCL, BJB, FF, SDS, AK, EEC, EMW, BAC, SEM, PJH, APE, DAD, AHK, HLE, JTY, KAM, ICD, KSC, KMM, BCT, CSF, DJS, RCT, EOL, AKS and GJM supported protocol development, refinement and implementation as directed by GHD and DRF BAC, SEM and PJH provided statistical expertise in clinical trial design and SEM is conducting the primary statistical analysis. DRF, DAT, LGK, DCL, BJB, FF, SDS, AK, EEC, EMW, BAC, SEM, PJH, APE, DAD, AHK, HLE, JTY, KAM, ICD, KSC, KMM, BCT, CSF, DJS, RCT, EOL, AKS and GJM reviewed and approved the final manuscript as led by GHD.

Funding Research reported in this work was funded through a Patient-Centered Outcomes Research Institute (PCORI) Award (1409-24099).

Disclaimer The views presented in this work are solely the responsibility of the author(s) and do not necessarily represent the views of PCORI, its board of governors or methodology committee.

\section{Competing interests None declared.}

Ethics approval This trial was approved by the University of Washington's Human Subjects Division on 21 April 2016. The University of Washington serves as the institutional review board (IRB) of record for the following study sites: University of Washington Medical Center, Harborview Medical Center, Virginia Mason Medical Center and Madigan Army Medical Center. Western IRB is the overseeing IRB for Swedish-First Hill (approved 8 July 2016) and Providence Regional Medical Center (approved 1 July 2016). UCLA-Olive View (approved 12 June 2016) and UCLAHarbor (approved 4 March 2016) are both regulated by their respective IRBs.

Provenance and peer review Not commissioned; externally peer reviewed.

Open Access This is an Open Access article distributed in accordance with the Creative Commons Attribution Non Commercial (CC BY-NC 4.0) license, which permits others to distribute, remix, adapt, build upon this work non-commercially, and license their derivative works on different terms, provided the original work is properly cited and the use is non-commercial. See: http://creativecommons.org/ licenses/by-nc/4.0/ 
(c) Article author(s) (or their employer(s) unless otherwise stated in the text of the article) 2017. All rights reserved. No commercial use is permitted unless otherwise expressly granted.

\section{REFERENCES}

1. Addiss DG, Shaffer N, Fowler BS, et al. The epidemiology of appendicitis and appendectomy in the United States. Am J Epidemiol 1990;132:910-25

2. Chang DC, Shiozawa A, Nguyen LL, et al. Cost of inpatient care and its association with hospital competition. J Am Coll Surg 2011;212:12-19.

3. Dieleman JL, Baral R, Birger M, et al. US spending on personal health care and public health, 1996-2013. JAMA 2016;316:2627-46.

4. Coldrey E. Five years of conservative treatment of acute appendicitis. $J$ Int Coll Surg 1959;32:255-61.

5. Wojciechowicz KH, Hoffkamp HJ, van Hulst RA. Conservative treatment of acute appendicitis: an overview. Int Marit Health 2010;62:265-72.

6. Styrud J, Eriksson S, Nilsson I, et al. Appendectomy versus antibiotic treatment in acute appendicitis. a prospective multicenter randomized controlled trial. World J Surg 2006;30:1033-7.

7. Hansson J, Körner U, Khorram-Manesh A, et al. Randomized clinical trial of antibiotic therapy versus appendicectomy as primary treatment of acute appendicitis in unselected patients. $\mathrm{Br} J$ Surg 2009:96:473-81.

8. Eriksson S, Granström L. Randomized controlled trial of appendicectomy versus antibiotic therapy for acute appendicitis. Br J Surg 1995;82:166-9.

9. Vons C, Barry C, Maitre S, et al. Amoxicillin plus clavulanic acid versus appendicectomy for treatment of acute uncomplicated appendicitis: an open-label, non-inferiority, randomised controlled trial. Lancet 2011;377:1573-9.

10. Mason RJ, Moazzez A, Sohn H, et al. Meta-analysis of randomized trials comparing antibiotic therapy with appendectomy for acute uncomplicated (no abscess or phlegmon) appendicitis. Surg Infect 2012;13:74-84

11. Findlay JM, Kafsi JE, Hammer C, et al. Nonoperative management of appendicitis in adults: a systematic review and meta-analysis of randomized controlled trials. J Am Coll Surg 2016;223:814-24.

12. Salminen $P$, Paajanen $H$, Rautio $T$, et al. Antibiotic therapy vs appendectomy for treatment of uncomplicated acute appendicitis: the APPAC randomized clinical trial. JAMA 2015;313:2340-8.

13. Ehlers AP, Talan DA, Moran GJ, et al. Evidence for an antibioticsfirst strategy for uncomplicated appendicitis in adults: a systematic review and gap analysis. J Am Coll Surg 2016;222:309-14.

14. Anderson JE, Bickler SW, Chang DC, et al. Examining a common disease with unknown etiology: trends in epidemiology and surgical management of appendicitis in California, 1995-2009. World J Surg 2012;36:2787-94.

15. Felson B. Appendical calculi; incidence and clinical significance. Surgery 1949;25:734-7.

16. Shindoh J, Niwa $\mathrm{H}$, Kawai $\mathrm{K}$, et al. Predictive factors for negative outcomes in initial non-operative management of suspected appendicitis. J Gastrointest Surg 2010;14:309-14.

17. Tanaka $Y$, Uchida $H$, Kawashima $H$, et al. Long-term outcomes of operative versus nonoperative treatment for uncomplicated appendicitis. J Pediatr Surg 2015;50:1893-7.

18. Gaskill CE, Simianu VV, Carnell J, Hippe DS, et al. Use of computed tomography to determine perforation in patients with acute appendicitis. Curr Probl Diagn Radiol 2016 (published Online First: December 7, 2016).

19. O'Leary DP, Lynch N, Clancy C, et al. International, expertbased, consensus statement regarding the management of acute diverticulitis. JAMA Surg 2015;150:899-904.

20. Vennix S, Morton DG, Hahnloser D, et al. Systematic review of evidence and consensus on diverticulitis: an analysis of national and international guidelines. Colorectal Dis 2014;16:866-78.

21. Morris AM, Regenbogen SE, Hardiman KM, et al. Sigmoid diverticulitis: a systematic review. JAMA 2014;311:287-97.
22. Talan DA, Saltzman DJ, Mower WR, Krishnadasan A, et al. Antibiotics-First versus surgery for appendicitis: a US pilot randomized controlled trial allowing outpatient antibiotic management. Ann Emerg Med 2017;70.

23. Ehlers AP, Davidson GH, Bizzell BJ, et al. Engaging stakeholders in surgical research: the design of a pragmatic clinical trial to study management of acute appendicitis. JAMA Surg 2016;151:580-2.

24. Harris PA, Taylor R, Thielke R, et al. Research electronic data capture (REDCap)-a metadata-driven methodology and workflow process for providing translational research informatics support. $J$ Biomed Inform 2009;42:377-81.

25. Solomkin JS, Mazuski JE, Bradley JS, et al. Diagnosis and management of complicated intra-abdominal infection in adults and children: guidelines by the Surgical Infection Society and the Infectious Diseases Society of America. Surg Infect 2010;11:79-109.

26. Singer M, Deutschman CS, Seymour CW, et al. The third International consensus definitions for sepsis and septic shock (Sepsis-3). JAMA 2016;315:801-10.

27. Le QA, Doctor JN, Zoellner LA, et al. Minimal clinically important differences for the EQ-5D and QWB-SA in Post-traumatic Stress Disorder (PTSD): results from a Doubly Randomized Preference Trial (DRPT). Health Qual Life Outcomes 2013;11:59.

28. Koumarelas K, Theodoropoulos GE, Spyropoulos BG, et al. A prospective longitudinal evaluation and affecting factors of health related quality of life after appendectomy. Int J Surg 2014;12:848-57.

29. ICH Harmonised Tripartite Guideline. Statistical principles for clinical trials. International conference on harmonisation E9 expert working group. Stat Med 1999;18-1905-42.

30. Piaggio G, Elbourne DR, Pocock SJ, et al. Reporting of noninferiority and equivalence randomized trials: extension of the CONSORT 2010 statement. JAMA 2012;308:2594-604.

31. Calvert M, Blazeby J, Altman DG, et al. Reporting of patient-reported outcomes in randomized trials: the CONSORT PRO extension. JAMA 2013;309:814-22.

32. Turner L, Shamseer L, Altman DG, et al. Consolidated standards of reporting trials (CONSORT) and the completeness of reporting of randomised controlled trials (RCTs) published in medical journals. Cochrane Database Syst Rev 2012;11:MR000030.

33. Angrist JD, Imbens GW. Two-stage least squares estimation of average causal effects in models with variable treatment intensity. J Am Stat Assoc 1995;90:431-42.

34. Little RJ, Yau LHY, Lhy Y. Statistical techniques for analyzing data from prevention trials: treatment of no-shows using Rubin's causal model. Psychol Methods 1998;3:147-59.

35. Bloom HS. Accounting for no-shows in experimental evaluation designs. Eval Rev 1984;8:225-46.

36. Diggle PJ HP, Liang KY, Zeger SL. Analysis of longitudinal data. Second Edition edn, 2002.

37. Turhan AN, Kapan S, Kutukcu E, et al. Comparison of operative and non operative management of acute appendicitis. Ulus Travma Acil Cerrahi Derg 2009;15:459-62.

38. Thorpe KE, Zwarenstein M, Oxman AD, et al. A pragmaticexplanatory continuum indicator summary (PRECIS): a tool to help trial designers. Can Med Assoc J 2009;180:E47-E57.

39. Khalil M, Rhee P, Jokar TO, et al. Antibiotics for appendicitis! Not so fast. J Trauma Acute Care Surg 2016;80:923-32.

40. Sitlani CM, Heagerty PJ, Blood EA, et al. Longitudinal structural mixed models for the analysis of surgical trials with noncompliance. Stat Med 2012;31:1738-60.

41. Detry MA, Lewis RJ. The intention-to-treat principle: how to assess the true effect of choosing a medical treatment. JAMA 2014;312:85-6.

42. Rabin R, de Charro F. EQ-5D: a measure of health status from the EuroQol Group. Ann Med 2001;33:337-43.

43. Amtmann D, Cook KF, Johnson KL, et al. The PROMIS initiative: involvement of rehabilitation stakeholders in development and examples of applications in rehabilitation research. Arch Phys Med Rehabil 2011;92:12-19.

44. Eypasch E, Williams JI, Wood-Dauphinee S, et al. Gastrointestinal quality of life index: development, validation and application of a new instrument. Br J Surg 1995;82:216-22.

45. Brehaut JC, O'Connor AM, Wood TJ, et al. Validation of a decision regret scale. Med Decis Making 2003;23:281-92. 